\title{
ENHANCEMENT OF VOIP PERFROMANCE IN MANET USING FUZZY LOGIC
}

\author{
Abdusalam Yahya, \\ Department of Computer Network, \\ College of Information Technology, \\ Elmergib University, Alkohms, Libye. \\ abdusalamabduallahyahya74@gmail.com \\ Ashraf Gasim Elsid Abdalla, \\ Faculty of Postgraduates studies, faculty of Telecommunication, \\ Future University, Khartoum, Sudan. \\ agea33@yahoo.com
}

Submitted: Aug, 07, 2020 Revised: Oct, 12, 2020 Accepted: Oct, 22, 2020

\begin{abstract}
Voiceover Internet Protocol (VoIP) application is a vital technology that is quickly growing in the Mobile Ad hoc NETwork (MANET). Packet loss is a factor that can significantly affect the Quality of Service (QoS) for VoIP performance. Due to the dynamic nature of MANET, it is a challenging task to maintain the desired packet loss rate. This paper aims to enhance the performance of VoIP in the MANET using a fuzzy logic model. The input for the model is VoIP packet loss and the outputs are the optimal parameters of MANET (node number, pause time, maximum speed, and maximum connection).Network Simulator (NS2) was used to perform all simulations. MATLAB was used to implement the proposed fuzzy model. Moreover, the performance of the model was evaluated using NS2, and the results show that our proposed fuzzy model offers a significant enhancement in terms of the VoIP packet loss rate (P.LR).
\end{abstract}

Keywords: VoIP, MANET, Fuzzy Logic.

\section{INTRODUCTION}

Voice over Internet Protocol (VoIP) is one of the fastest rising Internet applications in recent years[1].It replaces the old Public Switched Telephone Network (PSTN) with a converged network where data, voice, and video packets are carried together using a single IP-based network. The integration between old voice network (PSTN) and IP data network into one network offered low cost and improved network management. Originally networks were designed to provide services to non-real-time applications such as FTP, HTTP, and email services which can tolerate some delay, jitter, and packet loss. Therefore, they are not sensitive to low network performance.

The VoIP is a real-time application and it has special characteristics. It is unable to tolerate inefficient network performance. Thus, IP networks must afford exceptional services to carry voice packets [2]. VoIP is an application that employs Real-Time Transport Protocol (RTP) to transfer voice packets over IP based networks. RTP runs on top of the user datagram protocol (UDP), which is an unreliable delivery protocol. Regarding real-time communication, voice packets that delay in arrival at the destination are useless and equal to drop packets[3].

Public Switch Telephone Network (PSTN) has provided the requirements of businesses and consumers worldwide with telephone service for more than 100 years [4].PSTN can accommodate all voice quality requirements, as it was originally designed for transmitting voice signal. In contrast, wireless networks are an IP-based network that was originally designed to carry only data packets. 
Moreover, due to the mobility of the MANET network, congestion occurs and introduces a high packet loss rate. As a result, carrying voice packets over the MANET network requires much concern. The major VoIP quality metrics in the IP networks are Packet Loss, Jitter, and End-to-End Delay [5][6].

Fuzzy logic was invented by Dr. Lotfi Zadeh at the University of California at Berkeley in 1965. Fuzzy logic allows situations or problems to describe and processed in linguistic words such as "cold "or "hot" as an alternative to exact numeric values such as a degree or 40 degrees. The advantages of fuzzy logic such as a linguistic system led to adopting this technology in many fields such as electronic, engineering control, information processing, financial analysis, and much more [7].

Many researchers have proposed solutions for improving VoIP performance using different techniques. However, none of them has employed the VoIP packet loss rate as input for the fuzzy system. Therefore, the contribution of this paper is to build a new fuzzy logic system that considers packet loss rate as the input. The proposed system is used to predict the best parameters of MANET to minimize the VoIP packet loss rate. The input for the model is VoIP packet loss rate and outputs are MANET parameters (number of nodes numbers, maximum speed, maximum connection, and pause time).

The rest of this paper was organized as follows: In section 2, the related work was reviewed. Section3 discussed and illustrated the proposed approach. Section 4 presented the evaluation process. Section 5 discussed the results. Finally, the conclusion was discussed in section 6 .

\section{RELATED WORKS}

Using a Fuzzy logic-based approach for VoIP quality maintenance proposed in [8]. The authors employed the Resource reservation protocol (RSVP) to control the token bucket algorithm. They built the Fuzzy token system includes three variables (Bandwidth Rate, Buffer Size, and New Token bucket). Stability was used to determine the best method to get a new token bucket that does not cause any factors (Jitter - Delay - Packet loss). The result shows that whenever improving bandwidth rate and buffer size rate, a new token bucket at the same rate of improvement and vice versa [8].

The authors of [9] employed a multi-criteria decision-making scheme to evaluate and select the best network services. The decision-making algorithm is based on the fuzzy logic approach. The study includes five service providers in Turkey namely, ADSL, Fiber technology, Power Line Communication, and highspeed Internet access offered by Cable Television operators. Four user profiles were created from the target ISPs (profile1: video, profile2: VoIP, profile3: Streaming Media, profile4: Interactive). Their study concluded that the use of fuzzy logic led toa strong indication of the expert opinions on decision-making.

Sweta and Meen in [10] proposed a decision support system to control two issues in buffer management (drop decision when buffer overflow occurs and scheduling decision when a transmission chance starts). They suggested a development to the Customs Service time scheduling by integrating it within a fuzzy-based buffer ranking mechanism. The study was conducted in Delay Tolerant Networks (DTNs). The results show that the proposed Fuzzy logic based traffic differentiation technique reaches improved delivery performance over the existing traffic differentiation scheme for DTNs.

Demonstrating the use of fuzzy logic to control congestion in ATM and TCP/IP networks has been done In [11]. The researchers follow ATM Forum Traffic Management Specification version 4 to propose Fuzzy Explicit Rate Marking in ATM and TCP/IP best effort. They concluded that the proposed technique can offer important improvements in controlling congestion in computer networks. 
In [12] the authors presented a dynamic QoS solution for enterprise networks. The proposed decision-making is based on 0-order TSK fuzzy model. This technique was employed to modify the priority level of a packet in real-time by adjusting the value of Differentiated Services Code Point (DSCP) in the Internet Protocol (IP) header. The developed system was tested in a real network environment and the results show that the proposed system dynamically able to change data transmission priority for different situations to improve the quality of service.

Improving service quality and network performance were accomplished in[13]. The authors used the Sugeno fuzzy system and differential operation to optimize the output and input variables of the fuzzy controller. The results of the simulation show that the proposed algorithm is capable to control the queue more efficiently than classical techniques.

In [14] an Adaptive Neuro-Fuzzy Inference System proposed is proposed to identify congestion in the sink node. It enables the sink node to guess the congestion rate using local information like a participant, buffer occupancy, and traffic rate as input for the protocol. The results show that the proposed protocol can detect the congestion level correctly whenever there is a packet drop.

\section{IMPLEMENTATION OF FUZZY LOGIC}

Fuzzy logic can be implemented in several techniques, one of them is the Fuzzy Inference System (FIS) [15]. A fuzzy interference system is used to control the connection between the input and output variables of a system. The crisp inputs in the fuzzy inference system are changed into fuzzy inputs by using a fuzzification interface. After fuzzification, the rule base is used. Defuzzification is used to convert the fuzzy value to the real-life value which is the output.

\section{A. Traffic Generation}

The first step to build our model is to create input data. According to previous studies, NS2 is the most appropriate tool to simulate the dynamic nature of MANET [16].Therefore, to generate input data NS2 is used to build a MANET. VoIP traffic was modeled in ns2 by using Code Bit Rate (CBR) traffic. The Tool Command Language Script (TCLS) was used to write the TCL script. Table 1 illustrates simulation parameters designed to calculate the VoIP packet loss rate.

TABLE 1 Simulation Parameters

\begin{tabular}{|c|c|}
\hline Parameters & Values \\
\hline Simulation Area Size & 500 X500 m2 \\
\hline Pause Time (P.T) & $2-20$ \\
\hline Maximum Connection (M.C) & $2-20$ \\
\hline Maximum Speed (M.S) & $2-20$ \\
\hline NodeNumber(Node. No) & $5-40$ \\
\hline Traffic Type & CBR (VoIP) \\
\hline Simulation Time & $300 s e c$ \\
\hline Simulation runs & 18 \\
\hline
\end{tabular}

\section{B. VoIP Packet Loss Rate}

Packet loss arises once transmitted packets across a network never reach its destination [13]. The packet loss rate (P.L.R) is a significant parameter affecting 
the QoS of VoIP. However, it is expressed as a ratio and calculated using the following formula (((Number of sent packets - Number of received packets) / total sent packet) * 100) [17]. Table 2 shows the packet loss ratio of VOIP.

TABLE 2 Packet Loss ratio of VoIP

\begin{tabular}{|c|c|c|c|c|c|}
\hline & \multicolumn{6}{|c|}{ MANET Scenarios Parameters } & Performance Metric \\
\hline Scenario. No & Node & $\boldsymbol{P} . \boldsymbol{T}(\boldsymbol{s})$ & $\boldsymbol{M} . \boldsymbol{P}(\boldsymbol{M} / \mathbf{S})$ & $\boldsymbol{M} . \boldsymbol{C}$ & VoIP P.L.R (\%) \\
\hline 1 & 5 & 2 & 2 & 2 & 0.01 \\
\hline 2 & 8 & 4 & 4 & 4 & 0.02 \\
\hline 3 & 10 & 5 & 5 & 5 & 0.08 \\
\hline 4 & 12 & 6 & 6 & 6 & 0.19 \\
\hline 5 & 14 & 7 & 7 & 7 & 0.1 \\
\hline 6 & 16 & 8 & 8 & 8 & 0.55 \\
\hline 7 & 18 & 9 & 9 & 9 & 4.5 \\
\hline 8 & 20 & 10 & 10 & 10 & 6.5 \\
\hline 9 & 22 & 11 & 11 & 11 & 7.45 \\
\hline 10 & 24 & 12 & 12 & 12 & 11.5 \\
\hline 11 & 26 & 13 & 13 & 13 & 14.99 \\
\hline 12 & 28 & 14 & 14 & 14 & 20 \\
\hline 13 & 30 & 15 & 151 & 5 & 26.5 \\
\hline 14 & 32 & 16 & 16 & 16 & 39.16 \\
\hline 15 & 34 & 17 & 17 & 17 & 39.5 \\
\hline 16 & 36 & 18 & 18 & 18 & 40 \\
\hline 17 & 38 & 19 & 19 & 19 & 51.14 \\
\hline 18 & 40 & 20 & 20 & 20 & 52.06 \\
\hline
\end{tabular}

\section{Fuzzy System Construction}

The second step is to build a fuzzy system using MATLABR2016a. The input variable is the VoIP Packet loss rate and the output variables are optimum MANET parameters.

\section{Membership Function}

The membership function type for the input and outputs was Triangular membership that offered flexibility to represent the information. Table 3 shows the minimum and maximum value for the input and outputs membership functions. Figures 1 to 6 show a graphical representation of the membership functions.

\section{TABLE 3 Range of Fuzzy system Membership functions}

\begin{tabular}{|c|c|c|}
\hline Fuzzy Parameters & Minimum & Maximum \\
\hline Packet loss & 0 & 40 \\
\hline Node number & 2 & 20 \\
\hline Pause time $(\mathrm{sec})$ & 2 & 20 \\
\hline Max speed $(\mathrm{m} / \mathrm{s})$ & 2 & 20 \\
\hline Max connection & 2 & 20 \\
\hline
\end{tabular}


Figure 1 shows the triangular membership function of the VoIP packet loss ratio. The $\mathrm{x}$-axis represents the packet loss rate with a range from 0 to 40 , whereas the $y$-axis represents the degrees of membership in the $[0,1]$ interval. The membership function consists of five fuzzy sets (Very low (VL), Low (L), Just High $(\mathrm{JH})$, High $(\mathrm{H})$, and Very High $(\mathrm{VH}))$

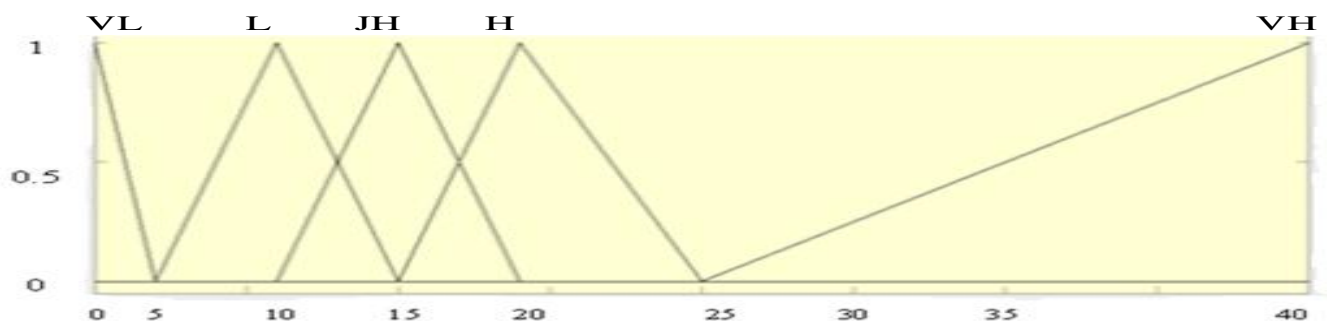

Fig. 1 Membership Function of VoIP Packet loss Ratio

Figure 2 displays the triangular membership function of the MANET node number. The $\mathrm{x}$-axis represents the number of nodes with a range from 2 to 20 , whereas the $y$-axis represents the degrees of membership in the $[0,1]$ interval. The membership function involves five fuzzy sets (Very low (VL), Low (L), Just High $(\mathrm{JH})$, High (H), and Very High (VH))

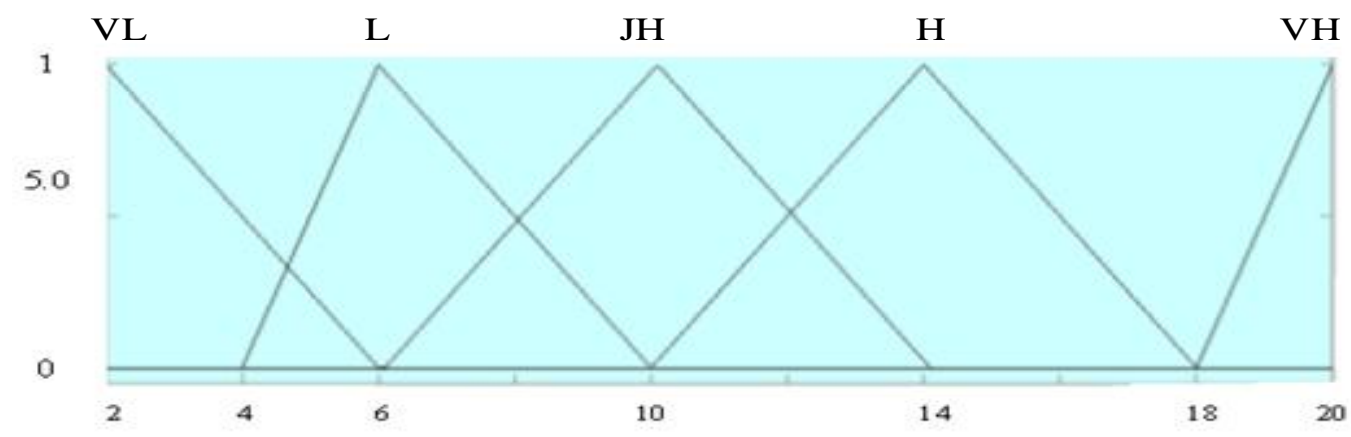

Fig. 2 Membership Function of MANET Number of nodes

Figure3 illustrates the triangular membership function of maximum speed (M. S) of MANET nodes. The $\mathrm{x}$-axis represents (M.S), whereas the $y$-axis represents the degrees of membership in the $[0,1]$ interval. The membership function consists of five fuzzy sets (Very low (VL), Low (L), Just High (JH), High (H), and Very High $(\mathrm{VH}))$

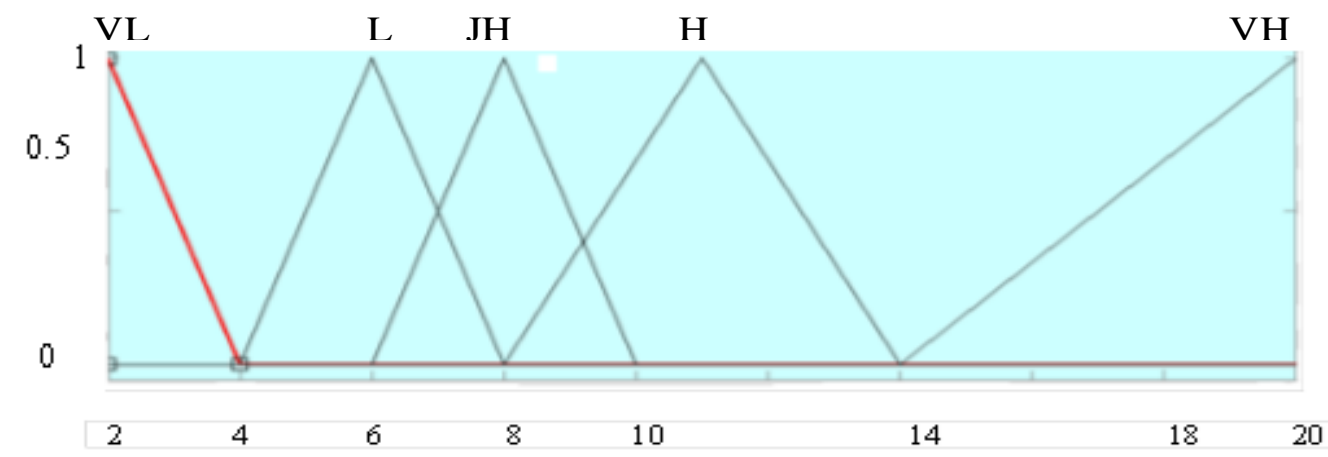

Fig. 3 Membership Function of Maximum speed 
Figure 4 plots triangular membership functions of maximum connection (M.C) in between MANET nodes. The x-axis represents the M.C range from 2to 20, whereas the $y$-axis represents the degrees of membership in the $[0,1]$ interval. The membership function contains five fuzzy sets (Very low (VL), Low (L), Just High (JH), High (H), and Very High(H))

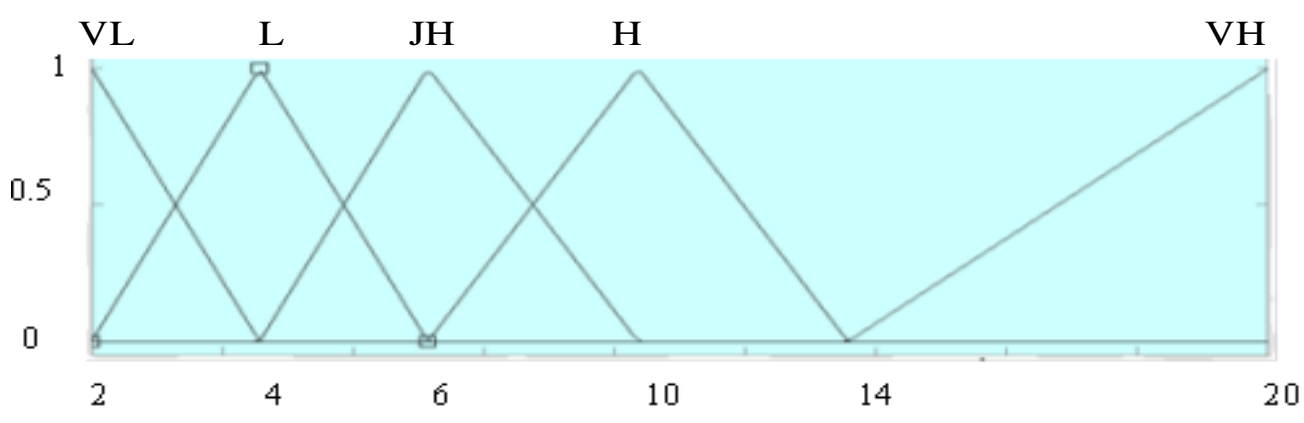

Fig. 4 Membership Function of Maximum Connection

Figure 5 presents the triangular membership functions of Pause time (P.T) of the mobility of MANET nodes. The x-axis represents the (P.T) with a range from 2 to 20 , whereas the $y$-axis represents the degrees of membership in the $[0,1]$ interval. The membership function includes five fuzzy sets (Very low (VL), Low (L), Just High (JH), High (H), and Very High (VH))

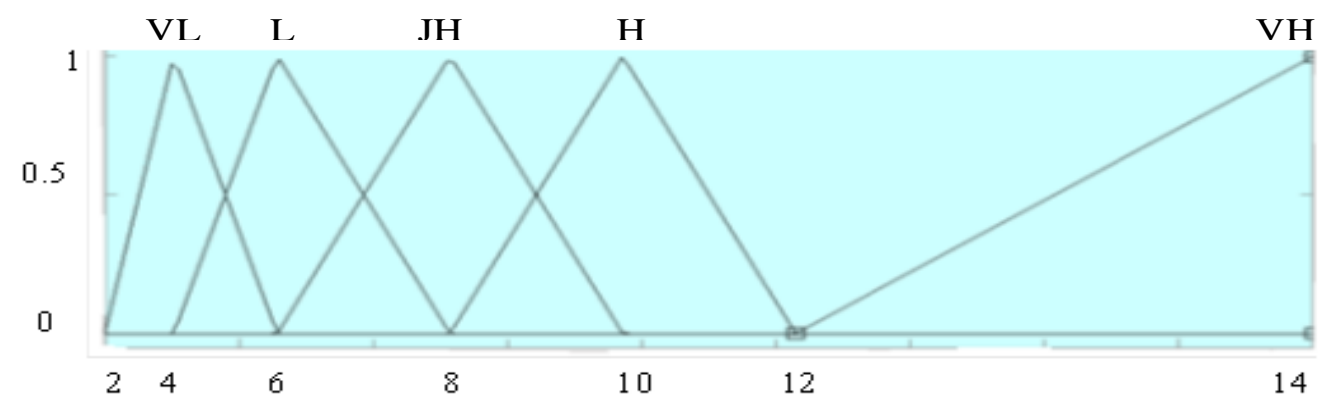

Fig. 5 Membership function of Pause time

\section{E. Fuzzy Rules}

The fuzzy logic system decides according to control rules and linguistic terms. In the main, the inference system has two models Mamdani and TakagiSugeno models. In this study, Mamdani approach is used because of its simple structure and straightforward design. The fuzzy rule consists of IF-THEN linguistic term and the output membership functions are worked by the fuzzy rule related between the input and the outputs. The suggested Fuzzy rules in this research are given in Table 4.

\section{PERFROMANCE EVALUATION}

There are no performance standards available to evaluate the goodness or accuracy of the fuzzy Membership function[18]. Therefore, the evaluation process is focused on the entire proposed fuzzy system. The following stages are performed 
to evaluate the model. Packet loss values stated in Table 2 are given as input to the model as shown in Figure 6.

TABLE 4 Rules used for the FIS knowledge base

\begin{tabular}{|c|c|c|c|c|c|c|}
\hline \multirow{6}{*}{$\mathrm{IF}$} & VoIP P.L.R & \multirow{6}{*}{ Then } & Node No. & P. T & M.C & M.S \\
\hline & Very Low & & Very Low & Very Low & Very Low & Very Low \\
\hline & Low (L) & & Low (L) & Low (L) & Low (L) & Low (L) \\
\hline & Just High & & Just High & Just High & Just High & Just High \\
\hline & High $(\mathrm{H})$ & & High $(\mathrm{H})$ & High $(\mathrm{H})$ & High $(\mathrm{H})$ & High $(\mathrm{H})$ \\
\hline & $\begin{array}{l}\text { Very High } \\
\text { (VH) }\end{array}$ & & $\begin{array}{c}\text { Very High } \\
\text { (VH) }\end{array}$ & $\begin{array}{l}\text { Very High } \\
\text { (VH) }\end{array}$ & $\begin{array}{l}\text { Very High } \\
\text { (VH) }\end{array}$ & $\begin{array}{l}\text { Very High } \\
\text { (VH) }\end{array}$ \\
\hline
\end{tabular}

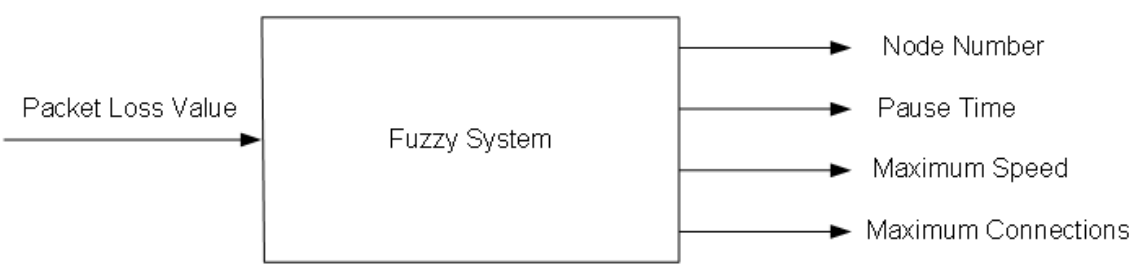

Fig. 6 Proposed Fuzzy System

The output from the fuzzy system in response to the input is illustrated in Table 5.

TABLE 5 Output of Proposed Fuzzy Mode

\begin{tabular}{|c|c|c|c|c|c|}
\hline Scenario. No & VoIP P.L.R(Input) & Nodes & M.S (m/s) & P.T (sec) & M.C \\
\hline 1 & 0.01 & 6 & 2 & 3 & 2 \\
\hline 2 & 0.02 & 6 & 2 & 3 & 2 \\
\hline 3 & 0.08 & 6 & 2 & 3 & 2 \\
\hline 4 & 0.19 & 6 & 2 & 3 & 2 \\
\hline 5 & 0.1 & 6 & 2 & 3 & 2 \\
\hline 6 & 0.55 & 6 & 2 & 3 & 2 \\
\hline 7 & 4.5 & 3 & 6 & 11 & 4 \\
\hline 8 & 6.5 & 5 & 6 & 7 & 5 \\
\hline 9 & 7.45 & 7 & 6 & 7 & 5 \\
\hline 10 & 11.5 & 11 & 9 & 8 & 8 \\
\hline 11 & 14.99 & 14 & 11 & 9 & 10 \\
\hline 12 & 20 & 11 & 11 & 11 & 11 \\
\hline 13 & 26.5 & 19 & 17 & 16 & 17 \\
\hline 14 & 39.16 & 19 & 18 & 17 & 17 \\
\hline 15 & 39.5 & 19 & 18 & 17 & 17 \\
\hline 16 & 40 & 19 & 18 & 17 & 17 \\
\hline 17 & 51.14 & 19 & 18 & 17 & 17 \\
\hline 18 & 52.06 & 19 & 18 & 17 & 17 \\
\hline
\end{tabular}

The model output values are used to set MANET scenario parameters as shown in Figure 7. The simulation is conducted using NS2 and VoIP packet loss 
ratio is calculated using AWK script language. Table 6 displays the result of run scenarios.

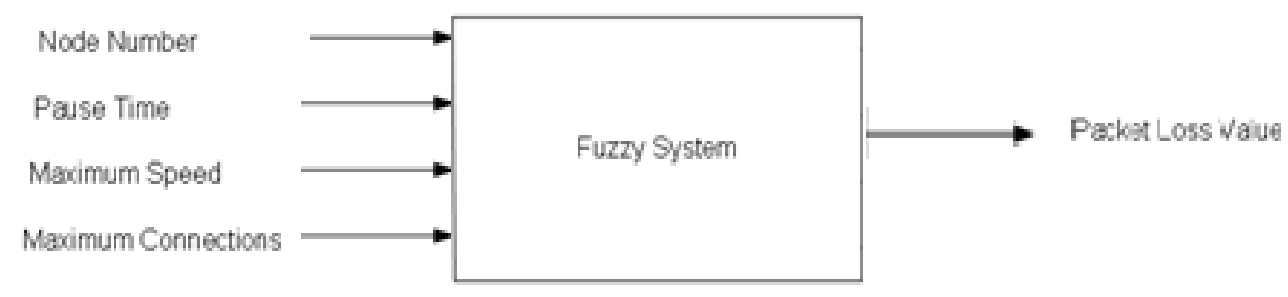

Fig. 7 Simulated Mobile ad-hoc Network

TABLE 6 Performance of VoIP with Fuzzy model

\begin{tabular}{|c|c|c|c|c|c|}
\hline Scenario. No. & Nodes & M.S (m/s) & P.T (sec) & M.C & VoIP P.L.R(\%) \\
\hline 1 & 6 & 2 & 3 & 2 & 0.02 \\
\hline 2 & 6 & 2 & 3 & 2 & 0.02 \\
\hline 3 & 6 & 2 & 3 & 2 & 0.02 \\
\hline 4 & 6 & 2 & 3 & 2 & 0.02 \\
\hline 5 & 6 & 2 & 3 & 2 & 0.02 \\
\hline 6 & 6 & 2 & 3 & 2 & 0.02 \\
\hline 7 & 3 & 6 & 11 & 4 & 0.65 \\
\hline 8 & 5 & 6 & 7 & 5 & 4 \\
\hline 9 & 7 & 6 & 7 & 5 & 6 \\
\hline 10 & 11 & 9 & 8 & 8 & 4.54 \\
\hline 11 & 14 & 11 & 9 & 10 & 12.71 \\
\hline 12 & 11 & 11 & 11 & 11 & 7.17 \\
\hline 13 & 19 & 17 & 16 & 17 & 25.5 \\
\hline 14 & 19 & 18 & 17 & 17 & 29.4 \\
\hline 15 & 19 & 18 & 17 & 17 & 29.4 \\
\hline 16 & 19 & 18 & 17 & 17 & 29.4 \\
\hline 17 & 19 & 18 & 17 & 17 & 35 \\
\hline 18 & 19 & 18 & 17 & 17 & 35 \\
\hline
\end{tabular}

\section{RESULTS AND DISCUSSION}

VoIP packet loss rate was calculated and compared with the proposed fuzzy model and without the model, as shown in Table 7 . The less VoIP packet loss rate was observed when using our model. This is due to applying fuzzy rules that produced the best MANET parameters. The result showed that the VoIP packet loss rate without fuzzy is between $0.01 \%$ and $51 \%$. In contrast, when the fuzzy model is used the rate is between $0.02 \%$ and $35 \%$ as shown in Table 7 . 
TABLE 7 Comparison of VoIP Packet loss ratio

\begin{tabular}{|c|c|c|}
\hline Scenario. No. & VoIP P.LR without Fuzzy (\%) & VoIP P.LR with fuzzy (\%) \\
\hline 1 & 0.01 & 0.02 \\
\hline 2 & 0.02 & 0.02 \\
\hline 3 & 0.08 & 0.02 \\
\hline 4 & 0.19 & 0.02 \\
\hline 5 & 0.1 & 0.02 \\
\hline 6 & 0.55 & 0.02 \\
\hline 7 & 4.5 & 0.65 \\
\hline 8 & 6.5 & 4 \\
\hline 9 & 7.45 & 6 \\
\hline 10 & 11.5 & 4.54 \\
\hline 11 & 14.99 & 12.71 \\
\hline 12 & 20 & 7.17 \\
\hline 13 & 26.5 & 25.5 \\
\hline 14 & 39.16 & 29.4 \\
\hline 15 & 39.5 & 29.4 \\
\hline 16 & 40 & 29.4 \\
\hline 17 & 51.14 & 35 \\
\hline 18 & 52.06 & 35 \\
\hline
\end{tabular}

\section{CONCLUSION}

In this paper, we employed a new fuzzy logic model to improve VoIP performance over MANET. The proposed system uses VoIP packet loss value to gain the ideal value of MANET parameters. The system is implemented in MATLAB R2016a.1.concerningtesting the model; we performed simulation experiments using NS2. The results approved that we significantly minimize the level of VoIP packet loss rate in mobile ad hoc networks.

\section{REFERENCES}

[1]. Y.-B. Lin and I. Chlamtac, Wireless, and mobile network architectures. John Wiley \& Sons, 2008.

[2]. S. Na and S. Yoo, "Allowable propagation delay for VoIP calls of acceptable quality," in International Workshop on Advanced Internet Services and Applications, 2002, pp. 47-55.

[3]. T.-K. Chua and D. C. J. I. N. Pheanis, "QoS evaluation of sender-based lossrecovery techniques for VoIP," Vol. 20, No. 6, 2006, pp. 14-22.

[4]. U. Varshney, A. Snow, M. McGivern, and C. J. C. o. t. A. Howard, "Voice over IP," Vol. 45, No. 1, 2002, pp. 89-96.

[5]. W. Wang, S. C. Liew, and V. O. J. I. t. o. v. t. Li, "Solutions to performance problems in VoIP over a 802.11 wireless LAN," Vol. 54, No. 1, 2005, pp. 366-384.

[6]. A. A. Yahya and M. A. Alhanish, "Study the Effect of OSPF and IS-IS Protocols Convergence on the VoIP Performance."

[7]. Y. Tanaka, "An overview of fuzzy logic," Proceedings of WESCON'93, 1993, pp. 446450 . 
[8]. M. E. Ebrahim and H. A. Hefny, "Fuzzy Logic based Approach for VoIP Quality Maintaining," International Journal Of Advanced Computer Science And Applications, Vol. 9, No. 1, 2018, pp. 537-542.

[9]. S. Balli and M. Tuker, "A fuzzy multi-criteria decision analysis approach for the evaluation of the network service providers in Turkey," Intelligent Automation \& Soft Computing, 2017, pp. 1-7.

[10]. S. Jain and M. Chawla, "A fuzzy logic based buffer management scheme with traffic differentiation support for delay tolerant networks," Telecommunication Systems, Vol. 68, No. 2, 2018, pp. 319-335.

[11]. K. Kumar, K. Narayana, and B. Sangmitra, "Congestion control in high speed networks using fuzzy logic control," International Journal of Mathematics and Soft Computing, Vol. 5, No. 1, 2015, pp. 45-55.

[12]. J. Li, L. Yang, X. Fu, F. Chao, and Y. Qu, "Dynamic QoS solution for enterprise networks using TSK fuzzy interpolation," IEEE International Conference on Fuzzy Systems, 2017, pp. 1-6.

[13]. N. K. Quoc, V. T. Tu, and N. T. Hai, "Some Improvements on Active Queue Management Mechanism Based on Adaptive Fuzzy Control," EAI Endorsed Transactions on Context-aware Systems \& Applications, Vol. 2, No. 6, 2015, p. e4.

[14]. T. Mekni, K. IbnTaarit, and M. Ksouri, "Adaptive neuro-fuzzy interence system congestioi detection protocol," International Conference on Advanced Systems and Electric Technologies, 2018, pp. 363-3680.

[15]. A. Salama, R. Saatchi, and D. Burke, "Fuzzy Logic and Regression Approaches for Adaptive Sampling of Multimedia Traffic in Wireless Computer Networks," Technologies, Vol. 6, No. 1, 2018, pp. 24.

[16]. T. P. Venkatesan, P. Rajakumar, and A. Pitchaikkannu, "Overview of Proactive Routing protocols in MANET," Fourth International Conference on Communication Systems and Network Technologies, 2014, pp. 173-177.

[17]. W. Mansouri, K. B. Ali, F. Zarai, and M. S. Obaidat, "Radio resource management for heterogeneous wireless networks: Schemes and simulation analysis," Modeling and Simulation of Computer Networks and Systems, 2015, pp. 767-792.

[18]. E. Kayacan, A. Sarabakha, S. Coupland, R. John, and M. A. J. E. A. o. A. I. Khanesar, "Type-2 fuzzy elliptic membership functions for modeling uncertainty," Vol. 70, 2018, pp. 170-183 